\title{
Amenazas, vulnerabilidad y riesgo de contaminación de las aguas subterráneas en la Península de Yucatán
}

DOI: $10.22403 /$ UQROOMX/TYP09/01

Francisco Bautista

Yameli Aguilar Duarte Centro de Investigaciones en Geografia Ambiental Universidad Nacional Autónoma de México

Eduardo Batllori Secretaría de Desarrollo Urbano y Medio Ambiente del Gobierno de Yucatán

\section{REsUmen}

En la Península de Yucatán (PY) el acuífero es la principal fuente de agua, pero es altamente vulnerable a la contaminación. Los objetivos del presente escrito son: I) realizar una reseña del seminario "Análisis de la vulnerabilidad y riesgo de contaminación de las aguas subterráneas en la Península de Yucatán"; 2) analizar el estado del arte en torno al manejo del agua subterránea en la región; y 3) elaborar algunas conclusiones generales que marquen las perspectivas a seguir. Entre las principales conclusiones se destaca que: a) la PY no es precisamente una "gran reserva de agua", $y$ es sólo un mito que es necesario romper; $b$ ) en la PY se tiene una gran cantidad y calidad de trabajos de disciplinas diferentes; c) el siguiente paso, que es menester dar a corto plazo, es la integración de estos estudios; d) el sector gubernamental promueve la necesidad de la formación de mesas de trabajo; y e) se enfatiza en la formación de nuevos recursos humanos con una visión integral.

\footnotetext{
Palabras Clima, contaminación natural y antrópica, relieve, riesgos CLAVE $\mid$ suelos.
}

Correos electrónicos: leptosol@ciga.unam.mx • eduardo.batllori@yucatan.gob.mx • yaguilar@pmip.unam.mx 


\section{Introducción}

En el nivel nacional se reconoce que los acuíferos de la Península de Yucatán (PY) son la gran reserva de agua dulce del país. En la PY, como en cualquier zona de karst, el acuífero es la principal fuente de abastecimiento para todas las actividades humanas. Para preservar la calidad del acuífero es necesario reconocer, estudiar y evaluar las amenazas (niveles y tipos de contaminantes), la vulnerabilidad (la protección natural del medio físico-biótico hacia el acuífero) y el riesgo de contaminación del acuífero (integración de las amenazas y la vulnerabilidad).

Las amenazas o peligros son la probabilidad de ocurrencia de un fenómeno potencialmente dañino; en este caso, corresponden a las actividades humanas y naturales que contaminan el acuífero. La vulnerabilidad es concebida como la potencial pérdida de la calidad del agua subterránea, debida al nivel de exposición natural de los acuíferos. El riesgo es el grado de pérdida de la calidad y cantidad de agua, por la ocurrencia de un suceso identificado previamente como amenaza; es una condición potencial de ocurrencia de algo nocivo o dañino que depende tanto de la intensidad de la amenaza, como de los niveles de vulnerabilidad del acuífero.

El estudio de las amenazas, la vulnerabilidad y el riesgo de contaminación de los acuíferos de la PY requiere una participación interdisciplinaria, es básicamente una actividad de equipo. Para lograr una visión más amplia sobre la problemática del manejo y protección del acuífero se organizó el seminario "Análisis de la vulnerabilidad y riesgo de contaminación de las aguas subterráneas en la Península de Yucatán". Sus objetivos fueron: I) ampliar y completar los equipos de trabajo que ya están organizados y en operación en bien de la conservación de los acuíferos peninsulares; 2 ) que los resultados del seminario sean de utilidad para los tomadores de decisiones en diversos niveles de gobierno; 3) finalmente, reunir a científicos y académicos de la región, para abrir un espacio de discusión sobre los retos y desafíos por enfrentar el manejo de las aguas subterráneas.

La idea de organizar el seminario nació durante la elaboración de un proyecto de investigación prácticamente con el mismo nombre, pero restringido al estado de Yucatán. Bautista y Aguilar (2006) se dieron cuenta de que: a) existen varios estudios aislados sobre diversos aspectos de la contaminación de las aguas subterráneas; b) los estudios sobre vulnerabilidad de las aguas subterráneas a la contaminación tienen diversos enfoques pero con debilidades 
cartográficas evidentes, como el uso de mapas integradores a partir de mapas de diferentes escalas, sin manejo de coordenadas, escala, ni rosa de vientos en los mapas, entre otras cosas; y c) los estudios de riesgo prácticamente son inexistentes, $y$, si los hay, no están publicados en revistas científicas indexadas (Bautista y Aguilar, 2006).

Los objetivos del presente escrito son: I) elaborar una reseña de lo ocurrido en el seminario; 2) análizar el estado del arte en torno al manejo del agua subterránea en la región; $y 3$ ) ofrecer algunas conclusiones generales que marquen las perspectivas a seguir.

\section{Los participantes}

Se recibieron 30 contribuciones relacionadas con el tema, y los participantes provinieron de diversos institutos y universidades del país y el extranjero: Facultad de Ingeniería, Facultad de Ingeniería Química, Facultad de Medicina Veterinaria y Zootecnia y Centro de Investigaciones Regionales Hideyo Noguchi, de la Universidad Autónoma de Yucatán (UADY); Centro de Investigación Científica de Yucatán (CICY) y su Unidad de Ciencias del Agua; Centro de Investigación y de Estudios Avanzados (Cinvestav, Unidad Mérida) del Instituto Politécnico Nacional; Centro de Ecología, Pesquerías y Oceanografía del Golfo de México (Epomex), de la Universidad Autónoma de Campeche (UAC); Comisión de Agua Potable y Alcantarillado de Chetumal; Universidad del Caribe; Instituto Tecnológico de Chetumal; Universidad de Quintana Roo (Uqroo); Instituto Nacional de Estadística y Geografía (INEGI) (Aguascalientes); Escuela de Ingeniería Ambiental de la Universidad de Guanajuato; Centro de Investigaciones en Geografía Ambiental (CIGA) (Michoacán), de la Universidad Nacional Autónoma de México (UNAM); Instituto de Geología, Facultad de Ingeniería, Facultad de Química, los tres de la UNAM; universidades canadienses, peruanas y españolas; Red de Malnutrición en Iberoamérica; Centro de Edafología y Biología Aplicada del Segura (CEBAS) e Instituto de Recursos Naturales y Agrobiología de Sevilla (IRNAS), ambos del Consejo Superior de Investigaciones Científicas (CSIC); así como consultores independientes Consultores en Agua Potable, Alcantarillado, Geohidrología \& Hidráulica Costera (CAPAGH). La gran mayoría de los trabajos recibidos tiene como zona de estudio el estado de Yucatán, seguido de Quintana Roo y por último Campeche. Fueron en total I 20 personas inscritas, con una participación aproximada de 100 personas por día. 
Fue evidente que hay un gran número de personas interesadas en el tema y que están realizando esfuerzos para entender los problemas actuales sobre el manejo y la protección de las aguas subterráneas en la PY y dilucidar los problemas futuros en escenarios probables, derivados del cambio climático global, del deficiente manejo y de la contaminación. Tanto las autoridades como los consultores independientes tienen un claro interés sobre el tema.

Este grupo de autoridades, investigadores, consultores y estudiantes, bien conducido y coordinado, podría ser de gran ayuda en la toma de decisiones en los niveles de gobierno municipal y estatal, así como en el nivel regional, pues la Península de Yucatán es una región geográfica.

\section{Las amenazas}

Las amenazas identificadas en el seminario fueron: I) la contaminación natural y antrópica; 2) la modificación de los flujos; 3) la sobreextracción de agua subterránea derivada del mito de que la PY es una gran reserva de agua; 4) el impacto del cambio climático global.

\section{La contaminación del agua subterránea}

La contaminación natural del agua subterránea se debe a la presencia de rocas del tipo de las evaporitas, que al solubilizarse se incorporan al agua, y por la natural intrusión salina en la zonas costeras; esto ocasiona su baja calidad tanto para consumo humano como para las actividades agrícolas. En este sentido, en la Península de Yucatán existen de manera general tres familias de aguas, la cálcicosulfatada en el sur, la sódica-clorurada en la costa, y la cálcico-carbonatada en la mayor parte de la Península; las dos primeras no son aptas para el consumo humano por las características de sus sales, y la cálcico-carbonatada representa la principal fuente de agua para los diferentes usos de la región.

En cuanto a la contaminación antrópica, los diversos contaminantes identificados en el agua subterránea son: plaguicidas, nitratos, coliformes fecales, hidrocarburos, metales pesados y productos de consumo humano como fármacos, cafeína, cocaína y nicotina (Gold, Metcalfe y Drovillard, 2009).

Los usos de suelo que más influyen en la contaminación de las aguas subterráneas son urbano industrial, urbano habitacional, agrícola con agroquímicos y pecuario con granjas de cerdos y aves. Dado que la descarga del 
acuífero llega a la costa, ha sido identificada como la causante de la eutrofización y la marea roja en la costa.

Un contaminante importante por su magnitud son las aguas residuales. A esta amenaza se le ha atendido en dos direcciones: a) el manejo de las aguas residuales de alta carga orgánica mediante la selección de los suelos que mejor funcionen como reactores naturales (descomposición y retención) o la aplicación a suelos en los que la materia orgánica se convierta en nutrimentos (Bautista et al., 2009); b) se propone el uso de residuos orgánicos, como la cáscara de la naranja, para el tratamiento de aguas residuales con colorantes (Cabañas y Vargas, 2009). En el nivel de investigación en desarrollo, cabe destacar el uso de viruta de hierro para cambiar los nitratos del agua en amonio.

Particularmente en las zonas urbanas, como Mérida y Cancún, se han encontrado contaminantes del tipo de los metales pesados, hidrocarburos, nitratos y amonio en el agua subterránea somera (Matthes, 2008; López, Macías, Cobos Gasca y Rendón von Osten, 2009; Pacheco y Cabrera, 2009), situación que muchas veces se ignora y, por lo tanto, no es atendida por las autoridades competentes.

En Yucatán, los pozos de abastecimiento de agua potable funcionan bien, igual que en Campeche, salvo excepciones. Esto es así porque los pozos de abastecimiento de agua potable extraen el agua del nivel profundo sin llegar a la salina; no obstante, la detección temprana de la contaminación de la lente de agua dulce que flota en la parte superior del agua salina subterránea es importante en la toma de decisiones en torno a la protección del acuífero.

Cuando se estudia la contaminación de las aguas subterráneas teniendo en mente las fuentes potenciales debe considerarse si la contaminación es difusa o puntual en la superficie, ya que el suelo cumple, entre otras, la función de amortiguar la contaminación, además de actuar como reactor natural para la descomposición o desactivación de los contaminantes. Sin embargo, cuando la capacidad amortiguadora del suelo se satura, éste se convierte en fuente de contaminación. Es por tales razones que las evaluaciones agroecológicas y agroambientales del uso de la tierra son de gran relevancia, como el modelo "pantanal" del sistema MicroLEIS (Díaz,Anaya-Romero y de la Rosa, 2009), que sirve para evaluar el riesgo real y potencial de contaminación de los suelos. Para atender esto se ha diseñado software que permite evaluar el uso de la tierra de manera sistemática y rápida $y$, por ende, una pronta y precisa toma de decisiones. 
En estudios espaciales se identificaron diversas áreas de oportunidad para mejorar el análisis y la representación cartográfica de las amenazas. Se recomienda diferenciar entre fuentes de contaminación fija y móvil, y entre tipo de contaminación puntual y difusa; identificar zonas de contaminación mediante mapas, ya sea por análisis estocástico o no estocástico; usar áreas buffer; emplear índices cuando se estudian varios elementos o indicadores, $y$ justificar matemáticamente las clases o intervalos de las leyendas de los mapas.

\section{Los flujos del agua subterránea}

El estudio de los flujos del agua subterránea es de vital importancia debido a que mediante él es posible conocer las áreas de recarga, las rutas de tránsito y sus velocidades, los destinos del agua y la identificación de las vías de la intrusión salina, entre otros aspectos.

En los estados de Yucatán y Quintana Roo se han realizado estudios sobre los modelos de los flujos del agua subterránea y se sabe que:

- El acuífero kárstico es una lente de agua dulce que flota sobre agua salada más densa (Perry,Velázquez-Olimán y Marín, 2002).

- La transmisividad del acuífero es muy elevada combinada con un gradiente hidráulico muy bajo que va de 7 a $10 \mathrm{~mm} \mathrm{~km}^{-1}$ a través de la mayor parte de la zona norte de la Península (Marín, et al., 1990; González-Herrera, Sánchez-y-Pinto y Gamboa Vargas, 2002).

- Los flujos de aguas subterráneas se dirigen hacia las zonas costeras, donde se presentan las descargas submarinas (Perry et al., 1995; González-Herrera, Sánchez-y-Pinto y Gamboa Vargas, 2002).

- El sistema acuífero recibe iones de dos fuentes naturales de gran magnitud, la disolución de minerales y la mezcla con la intrusión de agua de mar (Perry, Velázquez-Olimán y Marín, 2002).

- El espesor de la zona de transición es variable y no proporcional a la distancia al mar (Perry et al., 2009).

- Las aguas subterráneas están controladas por los flujos preferenciales debido a las estructuras de las cuevas.

Los flujos del agua subterránea no son bien conocidos, y se advierte que es necesario pasar del modelo global radial que corresponde a la escala regional 
(Península de Yucatán) a modelos con mayor detalle para cada estado y para cada zona en particular. En este aspecto se detecta un mayor desconocimiento, que podría verse como un área de oportunidad para realizar estudios y formar recursos humanos.

\section{La magnitud del agua subterránea}

Una característica importante de las aguas subterráneas de la Py es que, en gran proporción, el agua dulce aprovechable flota como una lente sobre agua salada densa que la subyace, aunado a que en su superficie se infiltran gran cantidad de contaminantes provenientes de los diversos usos industriales, pecuarios, agrícolas, de servicios y de uso público urbano. De esta manera, el agua dulce disponible podría presentar una gran reducción como consecuencia de las amenazas, la vulnerabilidad y el alto riesgo de contaminación.Además, hay que tener presente que el agua subterránea depende de la recarga pluvial y se ha detectado que en años secos la intrusión salina puede llegar a ser muy alta, por la baja presión que ejerce el agua subterránea en su camino de tierra adentro hacia el mar (Villasuso et al., 2009; Escolero, Jaime y Domínguez-Mariani, 2009).

Por ejemplo, en sus estadísticas de agua en México, la Comisión Nacional del Agua (Conagua, 2008) reportó para el caso de Yucatán que el volumen total concesionado y extraído de pozos alcanzó la cifra de II 02 millones de metros cúbicos, donde el sector agrícola pecuario y de acuacultura consumió 814 millones, el abastecimiento público urbano y doméstico alcanzó 245 millones de metros cúbicos, la industria 33 millones y las termoeléctricas y otras industrias y comercios casi diez millones. Esta extracción no afecta la disponibilidad actual y futura, pero las descargas de aguas residuales sí tienen la capacidad de repercutir en la disponibilidad del recurso. Los datos de la Conagua señalan para Yucatán vertimientos de 50 millones de metros cúbicos de aguas residuales de aguas industriales, acuícolas, porcícolas y de servicios, así como I 30 millones de metros cúbicos de aguas residuales del uso público urbano. Las cargas contaminantes orgánicas alcanzan la cifra de 157 toneladas diarias -podríamos mencionar también los lixiviados en basureros-. A pesar de que en los tres estados de la PY se tiene una estimación de la extracción y uso del agua subterránea, aún no se conoce la cantidad de agua subterránea que fuera sustentablemente extraída, es decir, una extracción de agua subterránea con la cual no se amenace la cantidad ni la calidad para las próximas generaciones (Escolero, Jaime y Domínguez Mariani, 2009). 


\section{El cambio climático global}

Un ascenso del nivel del mar, resultado del cambio climático global, provocaría una enorme intrusión salina y, por lo tanto, la salinización de grandes volúmenes de agua dulce y la inundación de una gran extensión del territorio (Ortiz y Méndez, 1999). Sin embargo, es necesario hacer investigación en detalle para documentar esta situación y cuantificar (con diferentes escenarios en número de centímetros de ascenso en el nivel del mar) hasta dónde llegaría la intrusión salina.

El incremento de la temperatura y, en consecuencia, de la evapotranspiración, así como la disminución de la precipitación pluvial, ocasionarían la reducción de la recarga del agua subterránea, lo que traería como resultado la disminución de la fuerza de los flujos de la tierra hacia el mar, por lo que es posible esperar un aumento de la intrusión salina.

\section{La vulnerabilidad}

La vulnerabilidad es un concepto que se refiere a la potencial pérdida de la calidad del agua subterránea debida al grado de exposición natural de los acuíferos. Los riesgos son la probabilidad de ocurrencia de algo nocivo o dañino, que depende tanto de la intensidad de la amenaza, como de los niveles de vulnerabilidad del acuífero.

Recientemente el concepto de vulnerabilidad ha incorporado los elementos de lugar, relaciones y patrones espaciales, y propone una metodología global para su evaluación denominada Spatial Analysis of the Vulnerability Environment (SAVE). Según este enfoque, la idea de lugar sirve para definir el área de estudio, la noción de relaciones espaciales es el principio que guía el análisis de las condiciones de vulnerabilidad, mientras que el concepto de patrón espacial provee el objetivo de la evaluación (Morales, 2009). Un estudio sobre la vulnerabilidad de las aguas subterráneas a la degradación debería también considerar la identificación de las zonas de reserva hidrológica (Escolero,Jaime y Domínguez-Mariani, 2009).

A pesar de la relevancia del estudio de la vulnerabilidad, sólo se han reportado trabajos de este tipo para el estado de Yucatán a escalas regionales y para la ciudad de Chetumal en el estado de Quintana Roo a escalas de detalle; desafortunadamente, no tienen reconocimiento oficial en los ordenamientos 
ecológicos del territorio de ambos estados.Al igual que en otras áreas de estudio, queda mucho por hacer en cuanto a la vulnerabilidad de la contaminación de las aguas subterráneas en la Península de Yucatán.

Respecto a los estudios de vulnerabilidad realizados en la región destacan los métodos GOD, DRASTIC Y EPIK. De éstos, únicamente el EPIK considera variables para acuíferos kársticos. El EPIK es el primer método de evaluación de vulnerabilidad para zonas kársticas diseñado por Doerfliger y Zwahlen en 1998. Por sus siglas en inglés, su nombre se deriva de los cuatro factores de evaluación que involucra: $\mathrm{E}$ (epikarst), $\mathrm{P}$ (protective cover), I (infiltration conditions) y K (karst network development). Este método basa su modelo conceptual de la vulnerabilidad en el tiempo de residencia -o velocidad de flujo- de un contaminante a través de los diferentes factores $\mathrm{P}, \mathrm{E}$ y $\mathrm{K}$ del acuífero kárstico, así como en la distinción entre el tipo de infiltración o entrada de agua al sistema (difusa o puntual), para definir los niveles de vulnerabilidad, que puede ser evaluada para un intervalo de escala de I:10 000 a 1:5 000; la cartografía final se obtiene por sobreposición y ponderación de los mapas monotemáticos (Doerfliger, Jeannin y Zwahlen, 1999; Goldscheider, 2005).Aunque el EPIK representa un avance metodológico, aún es complicada su aplicación, pues para el nivel de detalle para el cual está diseñado implicaría un gran esfuerzo obtener datos, lo cual se traduce en tiempo y costos.

Si se estuviera de acuerdo en que "la vulnerabilidad es la potencial pérdida de la calidad del agua subterránea debida al grado de exposición natural", entonces habría que analizar la función ambiental que cumple cada elemento del medio biofísico: I) el relieve es un factor que regula los flujos del agua superficial, los disipa o los concentra; 2) suelo y subsuelo son las capas protectoras y funcionan como filtros naturales debido a procesos como la retención y la descomposición; 3) el clima es la vía de transporte de los posibles contaminantes (la profundidad de la descarga o la intensidad de la lluvia); y 4) la hidrología, particularmente los flujos del agua subterránea, sirve para conocer el destino de los contaminantes. A continuación se realiza una descripción breve de cada elemento del medio biofísico.

\section{EI relieve kárstico}

El término karst se utiliza para referirse a un paisaje formado principalmente por la disolución de rocas solubles, como la caliza y la dolomita, que origina un conjunto diverso de topografías externas e internas, de formas singulares, 
a diferentes escalas y tamaños, desde muy pequeños como los microlapiaces, hasta depresiones cerradas, lomeríos, valles, cavernas y complejos sistemas de drenaje subterráneo con características hidrológicas particulares (EPA, 2002; Kueny y Day, 2002; de Waele, Plan y Audra 2009).

Esta gran variedad de formas del relieve de las zonas kársticas trae como consecuencia una alta heterogeneidad espacial y, por lo tanto, la especificidad para los usos del territorio (Bautista, 2008; deWaele, Plan y Audra, 2009). De tal modo, comprender los aspectos geomorfológicos es la base de la zonificación a escalas medianas y grandes (Bautista et al.,2005) y proporciona la base espacial para la delimitación de otros componentes del medio natural, útil para realizar ordenamientos ecológicos territoriales y elaborar instrumentos de política pública ambiental (Bocco, Mendoza y Velázquez, 200 I; Mendoza et al., 2009).

La PY es una plataforma con estratos de rocas carbonatadas; el clima dominante es de tipo cálido subhúmedo, lo que confiere a esta región un paisaje típico de karst tropical caracterizado por depresiones cerradas de diversas formas y tamaños, planicies y formas positivas como lomeríos y colinas. Los modelos digitales del terreno son de utilidad para la diferenciación geomorfológica en zonas con amplitud del relieve (Ihl et al., 2007), sin embargo, en aquellos sitios donde predominan las grandes planicies, es necesario considerar otros atributos del relieve para la zonificación geomorfológica. Las depresiones kársticas son rasgos característicos del paisaje que pueden ser reconocidas en mapas topográficos I:50 000 (Frausto e Ihl, 2008); su abundancia y tamaño son indicadores de la intensidad de la disolución de la roca, del desarrollo kárstico y de los flujos preferenciales del agua subterránea (White y White, 1979).

Se han reportado diversos trabajos geomorfológicos que utilizan la morfometría y el análisis de la distribución de parámetros de depresiones kársticas para el estudio de la karstificación (White yWhite, 1979; Shofner, Mills y Duke, 200I; Denizman, 2003; Florea, 2005). La densidad de depresiones ha sido una de las técnicas más comunes para medir su abundancia; la aplicación de técnicas morfométricas proporciona un análisis objetivo y cuantitativo del sistema kárstico para su descripción y análisis geográfico (Denizman, 2003), además de que apoyaría el reconocimiento de las zonas con mayor concentración de flujo o recarga puntual de agua y contaminantes, y por lo tanto servirían como base cartográfica en el análisis de la vulnerabilidad. 


\section{Suelos}

Los suelos poseen una capacidad natural para amortiguar el paso de los contaminantes y depurar el agua (Bautista, Durán de Bazúa y Lozano, 2000; Bouma, 2006).Algunas propiedades edáficas que influyen significativamente en la capacidad de amortiguamiento son: capacidad de intercambio de cationes, arcillas, materia orgánica, $\mathrm{pH}$ y carbonatos. Por esta razón se debe considerar este elemento del ambiente en el análisis de la vulnerabilidad. Sin embargo, la investigación edafológica en la PY es incipiente.

Es menester publicar los nuevos mapas de suelos de la Península deYucatán utilizando la clasificación internacional de suelos (WRB, 2006) y los mapas estatales, al menos a escalas 1:250 000, o de preferencia a escala l:50 000 . De manera general se piensa que el suelo es altamente permeable, pero esta aseveración sólo corresponde a Leptosoles y Arenosoles, en los demás grupos de suelo no es así, incluso hay Vertisoles de baja velocidad de infiltración, así como Gleysoles y Stagnosoles de drenaje impedido. No obstante, los estudios al respecto son muy escasos.

En la última década se han generado modelos matemáticos denominados funciones de pedotransferencia (FPT), los cuales se basan en emplear propiedades del suelo ampliamente conocidas y reportadas en los levantamientos de suelos para estimar procesos de difícil medición, sobre todo de las propiedades hidráulicas del suelo (cuadro I). La utilización de las FPT fue reconocida de inmediato por su gran variedad de usos; por ejemplo, la retención de humedad y los parámetros de transporte son usados en hidrología para saber la proporción de agua de infiltración y escorrentía de acuerdo con la precipitación pluvial (Bouma, 1 989; Wösten, 2002; Pachepsky, Rawls y Lin, 2006).

Se requiere conocer la velocidad de infiltración del agua a través de los suelos, o al menos generar ecuaciones de edafotransferencia (pedotransferencia) para cada grupo de suelo, por ejemplo Cambisoles, Luvisoles, Stagnosoles, Gleysoles, Leptosoles, etcétera.

\section{Subsuelo}

Una forma de estimar el poder protector del subsuelo es conocer su espesor a partir del modelo digital de elevación y el mapa altimétrico, considerando que a mayor espesor del subsuelo menor vulnerabilidad. Marín, Pacheco y 
Amenazas, vulnerabilidad y riesgo de contaminación de las aguas subterráneas en la Península de Yucatán

CUADRO I. FPT GENERADAS PARA ESTIMAR CONDUCTIVIDAD HIDRÁULICA EN SUELOS

\begin{tabular}{|c|c|c|c|}
\hline Autor & Ecuación & Variables & Referencia \\
\hline $\begin{array}{l}\text { Vereecken et al., } \\
1990\end{array}$ & $\begin{array}{l}\ln \left(K_{s}\right)=20.62-0.96 * \ln (C L)-0.66 * \ln (S A)- \\
0.46 * \ln (M O)-8.43 * D_{b}\end{array}$ & $\begin{array}{l}C L=\text { arcilla; } S A=\text { arena; } \\
M O=\text { materia orgánica en } \\
(\%) ; D_{b}=\text { densidad aparente } \\
\left(\mathrm{g} \mathrm{cm}^{-3}\right)\end{array}$ & $\begin{array}{l}\text { Nemes et al., } \\
2005\end{array}$ \\
\hline $\begin{array}{l}\text { Vereecken et al., } \\
1990\end{array}$ & $\begin{array}{l}\ln \left(K_{s}\right)=\mid 8.096-0.324 * f I+0.312 * f 2- \\
0.305 * f 3+0.363 * f 5+0.370 * f 6-0.774 * M O- \\
9.056 * D_{b}\end{array}$ & $\begin{array}{l}\text { Simbología igual que la } \\
\text { anterior. Detalles de la } \\
\text { distribución de tamaño de } \\
\text { partículas (fl a f6) }\end{array}$ & $\begin{array}{l}\text { Nemes et al., } \\
2005\end{array}$ \\
\hline $\begin{array}{l}\text { Wösten et al., } \\
1999\end{array}$ & $\begin{array}{l}\ln \left(K_{s}\right)=7.75+0.0352 * S I+0.93 *(T O P S O I L)- \\
0.967 * D_{b}^{2}-0.000484 * \mathrm{CL}^{2}- \\
0.000322 * \mathrm{SI}^{2}+0.00 I^{2} \mathrm{SI}^{-1}-0.0748 * \mathrm{MO}- \\
{ }^{\mathrm{I}}-0.0643 * \ln (\mathrm{SI})-0.01398 * \mathrm{D}_{\mathrm{b}}^{*} \mathrm{CL}- \\
0.1673 * \mathrm{D}_{\mathrm{b}} * \mathrm{OM}+0.02986 *(\mathrm{TOPSOIL}) * \mathrm{CL}- \\
0.03305 *(\mathrm{TOPSOIL}) * \mathrm{SI}\end{array}$ & $\begin{array}{l}\mathrm{CL}=\text { arcilla; } \mathrm{SA}=\text { arena; } \\
\mathrm{SI}=\text { limo; } \mathrm{OM}=\text { materia } \\
\text { orgánica en }(\%) ; \mathrm{D}_{\mathrm{b}}=\text { densidad } \\
\text { aparente; TOPSOIL como } \\
\text { variable categórica: I si la } \\
\text { muestra de suelo proviene } \\
\text { de la capa superior del suelo } \\
\text { (ej. Horizontes A o E de } \\
\text { acuerdo a la clasificación } \\
\text { FAO, I990) ó } 0 \text { si la muestra } \\
\text { proviene del subsuelo }\end{array}$ & $\begin{array}{l}\text { Nemes et al., } \\
\text { 2005; Wösten } \\
\text { et al., I999:; } \\
\text { Wösten et al., } \\
2001 .\end{array}$ \\
\hline
\end{tabular}

\begin{tabular}{|c|c|c|c|}
\hline $\begin{array}{l}\text { Wösten et al., } \\
200 \text { I }\end{array}$ & $\begin{array}{l}\ln \left(\mathrm{K}_{\mathrm{s}}\right)=\quad 45.8-\left|4.34 * \mathrm{D}_{\mathrm{b}}+0.00\right| 48 \mid * \mathrm{SI}^{2}{ }_{-} \\
27.5 * \mathrm{Db}-\mathrm{I}-0.89 \mid * \ln (\mathrm{SI})-0.34 * \ln (\mathrm{MO})\end{array}$ & $\begin{array}{l}C L=\text { arcilla; } S A=\text { arena; } \\
M O=\text { materia orgánica en }(\%) ; \\
D_{b}=\text { densidad aparente }\end{array}$ & $\begin{array}{l}\text { Nemes et al., } \\
2005\end{array}$ \\
\hline $\begin{array}{l}\text { Wösten et al., } \\
200 \text { I }\end{array}$ & $\begin{array}{l}\ln \left(K_{s}\right)=-42.6+8.7 I * M O+6 I .9 * D_{b}- \\
20.79 * D^{2}-0.2107 * M^{2}-0.01622 * C L * M O- \\
5.382 * D_{b} * M O\end{array}$ & $\begin{array}{l}\mathrm{CL}=\text { arcilla; } \mathrm{SA}=\text { arena; } \\
\mathrm{MO}=\text { materia orgánica en }(\%) ; \\
\mathrm{Db}=\text { densidad aparente }\end{array}$ & $\begin{array}{l}\text { Nemes et al., } \\
2005\end{array}$ \\
\hline $\begin{array}{l}\text { Brankensiek } \\
\text { et al., } 1984\end{array}$ & $\begin{array}{l}\mathrm{K}_{\mathrm{s}}(\mathrm{mm} / \mathrm{h})=10 \exp [19.5 \phi-8.97- \\
0.028212\left(\mathrm{P}_{<2}\right)+0.00018107\left(\mathrm{P}_{50-}\right. \\
2000)^{2}-0.0094125\left(\mathrm{P}_{<2}\right)^{2}-8.395215 \\
\phi^{2}+0.077718\left(\mathrm{P}_{50-2000}\right) \phi-0.00298\left(\mathrm{P}_{50-2000}\right)^{2} \\
\phi^{2}-0.019492\left(\mathrm{P}_{<2}\right)^{2} \phi^{2}+0.0000173\left(\mathrm{P}_{50-}\right. \\
2000) 2\left(\mathrm{P}_{<2}\right)+0.02733\left(\mathrm{P}_{<2}\right)^{2} \\
\phi+0.001434\left(\mathrm{P}_{50-2000}\right)^{2} \\
\left.\phi-0.0000035\left(\mathrm{P}_{<2}\right)^{2}\left(\mathrm{P}_{50-2000}\right)\right]\end{array}$ & $\begin{array}{l}\mathrm{P}_{<2}=\text { arcilla; } \mathrm{P}_{2-50}=\text { limo; } \\
\mathrm{P}_{>50}=\text { arena } ; \mathrm{BD}=\text { densidad } \\
\text { aparente; } \phi=\text { porosidad }\end{array}$ & $\begin{array}{l}\text { Sobieraj et al., } \\
2001\end{array}$ \\
\hline $\begin{array}{l}\text { Campbell and } \\
\text { Shiozawa } 1994\end{array}$ & $\mathrm{~K}_{\mathrm{s}}(\mathrm{mm} / \mathrm{h})=54 \exp \left[-0.07 \mathrm{P}_{50-2000}-0.167 \mathrm{P}_{<2}\right]$ & $\begin{array}{l}\mathrm{P}_{<2}=\operatorname{arcilla} ; \mathrm{P}_{2-50}=\operatorname{limo} ; \mathrm{P}_{>50}= \\
\text { arena }\end{array}$ & $\begin{array}{l}\text { Sobieraj et al., } \\
200 \mathrm{I}\end{array}$ \\
\hline
\end{tabular}


CUADRO I. FPT GENERADAS PARA ESTIMAR CONDUCTIVIDAD HIDRÁULICA EN SUELOS

\begin{tabular}{|c|c|c|c|}
\hline Autor & Ecuación & Variables & Referencia \\
\hline $\begin{array}{l}\text { Cosby et al., } \\
1984\end{array}$ & $\begin{array}{l}K_{s}=(\mathrm{mm} / \mathrm{h})=25.4 \times 10\left(-0.6+0.012 P_{50-2000}\right. \\
\left.-0.0064 \mathrm{P}_{<2}\right)\end{array}$ & $\begin{array}{l}P_{<2}=\text { arcilla } ; P_{2-50}=\operatorname{limo} ; P_{>50}= \\
\text { arena }\end{array}$ & $\begin{array}{l}\text { Sobieraj et al., } \\
2001\end{array}$ \\
\hline Jabro 1992 & $\begin{array}{l}\log \left(K_{s}\right)(\mathrm{cm} / \mathrm{h})=9.56-0.81 \log \left(P_{2-50}\right)-1.09 \\
\log \left(P_{<2}\right)-4.64(B D)\end{array}$ & $\begin{array}{l}\mathrm{P}_{<2}=\text { arcilla } ; P_{2-50}=\text { limo; } \\
\mathrm{P}_{>50}=\text { arena } ; \mathrm{BD}=\text { densidad } \\
\text { aparente }\end{array}$ & $\begin{array}{l}\text { Sobieraj et al., } \\
2001\end{array}$ \\
\hline $\begin{array}{l}\text { Puckett et al., } \\
1985\end{array}$ & $\mathrm{~K}_{\mathrm{s}}(\mathrm{mm} / \mathrm{h})=156.96 \exp \left[-0.1975 \mathrm{P}_{<2}\right]$ & $P_{<2}=$ arcilla & $\begin{array}{l}\text { Sobieraj et al., } \\
2001\end{array}$ \\
\hline $\begin{array}{l}\text { Dane and } \\
\text { Puckett } 1994\end{array}$ & $K_{s}(\mathrm{~mm} / \mathrm{h})=303.84 \exp \left(-0.144 \mathrm{P}_{<_{2}}\right)$ & $\mathrm{P}_{<2}=$ arcilla & $\begin{array}{l}\text { Sobieraj et al., } \\
2001\end{array}$ \\
\hline $\begin{array}{l}\text { Saxton et al., } \\
1986\end{array}$ & $\begin{array}{l}\mathrm{K}_{\mathrm{s}}(\mathrm{mm} / \mathrm{h})=10 \exp \left[\mathrm{I} 2.012-0.0755 \mathrm{P}_{50-}\right. \\
20000\left(-3.895+0.0367 \mathrm{IP} \mathrm{P}_{50-2000^{-}}\right. \\
\left.0.1103 \mathrm{P}_{<<}+0.00087546\left(\mathrm{P}_{<2}\right) 2 / \theta_{\mathrm{s}}\right], \text { donde } \\
\theta_{\mathrm{s}}=0.332-0.000725 I \mathrm{P}_{50-2000}+0.1276 \\
\log \mid 0\left(\mathrm{P}_{<2}\right)\end{array}$ & $\begin{array}{l}P_{<2}=\text { arcilla; } P_{2-50}=\text { limo; } \\
P_{>50}=\text { arena; } \theta_{s}=\text { contenido } \\
\text { saturado de humedad }\end{array}$ & $\begin{array}{l}\text { Sobieraj et al., } \\
2001\end{array}$ \\
\hline
\end{tabular}

Méndez (2004) han apreciado que hay una diferencia de sólo tres metros entre el nivel piezométrico y la altitud al nivel medio del mar en las planicies kársticas del estado de Yucatán. Los estudios sobre la velocidad de infiltración en el subsuelo, así como su capacidad buffer ante contaminantes, son muy escasos (véase cuadro I).

\section{El clima}

No hubo ponencias sobre el clima, a pesar de que la lluvia es el vehículo por el cual se transportan los contaminantes de la superficie del suelo al agua subterránea. Hay diversas posibilidades de incluir el clima en estudios de vulnerabilidad, desde el tradicional uso de la precipitación total anual o el índice de humedad anual $(\mathrm{IH})$, el índice de aridez, y el índice de concentración de las precipitaciones, que son muy generales, hasta las muy particulares como el índice de lavado de suelos (ILS) (Oliver, 1980; de la Rosa et al., 1996):

- Índice de humedad. Este índice anual se utiliza para estimar, de una forma general, el agua disponible para las plantas.También se suele emplear para 
prever las necesidades de drenaje artificial en una zona. Para calcular el índice de humedad (HUi) se aplica la siguiente fórmula:

$\mathrm{HUi}=\mathrm{P} / \mathrm{ETO}$

Donde: $\mathrm{P}=$ precipitación; $\mathrm{ETo}=$ evapotranspiración potencial

El valor de este índice va de $<0.05$ hasta > I, con seis categorías: hiperáridas, áridas, semiáridas, subhúmedas secas, subhúmedas húmedas y húmedas, respectivamente (Lobo et al., 2004).

- Índice de aridez. Como un índice anual, este procedimiento simple trata de estimar la aridez general del clima. El índice de aridez (ARi) se calcula en función del número de meses del año en que la evapotranspiración potencial excede a la precipitación.

- Índice de concentración de las precipitaciones. Con el fin de estimar la agresividad de las lluvias, a partir de la variabilidad temporal de las precipitaciones mensuales, Oliver (1980) propuso el índice de concentración de las precipitaciones ( $\mathrm{PCi})$, expresado en porcentaje mediante la siguiente fórmula:

$\mathrm{PCi}=100 \times \sum\left(\mathrm{P}^{2} / \mathrm{P}^{2}\right)$

Donde: $\mathrm{P}=$ precipitación mensual; $\mathrm{P}=$ precipitación anual

Este índice, cuyo valor oscila entre 8.3 y $100 \%$, parece ser una adecuada expresión estadística para comparar la concentración de las lluvias entre estaciones.Así, un índice bajo equivale a una distribución uniforme de las lluvias, mientras que un índice alto corresponde a una elevada concentración de ellas.

- Índice de Arkley. El índice de Arkley (AKi) se utiliza para estimar el efecto del clima sobre el proceso de lavado en los suelos.Arkley (de la Rosa et al., 1996) definió este índice anual como el valor más elevado bien de la suma de las precipitaciones mensuales menos las evapotranspiraciones potenciales de aquellos meses en que la precipitación es mayor que la evapotranspiración, o bien de la cantidad total de precipitación del mes más húmedo. Los intervalos del índice de lavado de suelos son: $<300,300-500$ y $>500$, para categorías de baja, moderada y elevada, respectivamente. 
Como en diversos índices climáticos se usa el valor de la evapotranspiración, el cálculo de la evapotranspiración potencial (ETo) debe ser realizado con base en estudios previos sobre la calibración de las ecuaciones de Hargreaves y Thornthwaite con la ecuación de Penman para estimar la ETo (Borges y Mendiondo, 2007; Bautista, Bautista y Delgado-Carranza 2009). El conocimiento preciso sobre la ETo tiene diversas ventajas, entre las que destacan una mejor estimación de la recarga y un mejor balance hídrico (Xu y Chen, 2005; Xu y Singh, 2005).

Para tener en cuenta la variación intraanual se recomiendan las siguientes opciones:

- La longitud del periodo de crecimiento (LPC), utilizando la P y la ETP. El inicio se determina cuando la $P$ es mayor que 0.5 de la ETp, el término del periodo es cuando la $\mathrm{P}$ es menor que 0.5 de la ETp. La unidad de contabilización de la lpc es en meses.

- La duración del periodo de lluvia que se puede identificar con el índice de humedad mensual, para identificar los meses húmedos a lo largo del año.

En el caso de existir una clara estacionalidad se recomienda el uso de modelos de vulnerabilidad por estación del año. De la misma manera, si se cuenta con un amplio registro de años es importante calcular la desviación estándar en el periodo de registro de los datos a utilizar. Finalmente, para integrar el análisis de vulnerabilidad, el cuadro 2 muestra un ejemplo de las variables que hay que considerar dependiendo de la escala de estudio.

Cuadro 2. Elementos del ambiente relacionados de ACuerdo

CON TRES ESCALAS DE MAPEO

\begin{tabular}{|c|c|c|c|}
\hline Elemento/escala & $\mathrm{I}: 250000$ & $\mathrm{I}: 50000$ & $\mathrm{I}: 25000$ \\
\hline Relieve & Geoformas & $\begin{array}{l}\text { Superficie de } \\
\text { depresiones }\end{array}$ & $\begin{array}{l}\text { Tipo de depresiones } \\
\text { (Cenotes, dolinas, uvalas y } \\
\text { poljés) }\end{array}$ \\
\hline Suelos & Asociación & Grupo & $\begin{array}{l}\text { Grupo y calificadores } \\
\text { primarios }\end{array}$ \\
\hline Subsuelo & $\begin{array}{l}\text { Altitud al nivel medio } \\
\text { del mar }\end{array}$ & $\begin{array}{l}\text { Nivel } \\
\text { piezométrico }\end{array}$ & $\begin{array}{l}\text { Nivel piezométrico y Tipo } \\
\text { de subsuelo }\end{array}$ \\
\hline Clima & $\begin{array}{l}\text { Duración del periodo } \\
\text { de lluvia } \\
\text { Índice de concentración } \\
\text { de la precipitación }\end{array}$ & & \\
\hline
\end{tabular}




\section{EI riesgo}

En este caso en particular, el riesgo es el grado de pérdida de la calidad o cantidad potencial de agua subterránea debido a las amenazas de contaminación, a la modificación de los flujos, a la sobreextracción y al cambio climático, etc., y depende también de los niveles de vulnerabilidad.

Para plasmar el estudio de riesgo en un mapa, es necesario, primero, hacer un mapa adecuado de la amenaza, el cual deberá considerar el tamaño de la fuente generadora del contaminante, la superficie que abarca el contaminante (incluyendo un área buffer) y el área administrativa (localidad, municipio, estado, región). Es posible utilizar intervalos en el caso de un índice o clases. Para seleccionar la cantidad de intervalos es recomendable un número impar, tres, cinco, siete. Para corroborar el uso de los intervalos de clase se sugiere elaborar un histograma para elegirlos, y posteriormente realizar un árbol de decisión para clasificación con el fin de comprobar que los intervalos hayan sido bien seleccionados.

CuAdro 3. EJeMPLO dE MANEJO CARTOGRÁfiCo INTEGRANDO LAS CLASES DE VULNERABILIDAD Y AMENAZA EN ESTUDIOS DE RIESGO

\begin{tabular}{lcccl}
\hline Categorías & Amenaza & Vulnerabilidad & Suma & Riesgo \\
\hline Muy alto & 5 & 5 & 9 a 10 & Muy alto \\
Alto & 4 & 4 & 7 a 8 & Alto \\
Medio & 3 & 3 & 5 a 6 & Medio \\
Bajo & 2 & 2 & 3 a 4 & Bajo \\
Muy Bajo & $\mathrm{I}$ & $\mathrm{I}$ & $\mathrm{I}$ a 2 & Muy Bajo \\
Nulo & 0 & 0 & Cuando una es 0 & Nulo
\end{tabular}

De la misma manera, el mapa de la vulnerabilidad deber ser cotejado mediante un árbol de decisión para clasificación. También se recomienda el empleo de un número impar en las clases.

Una vez que se cuenta con los dos mapas, uno de amenazas y otro de vulnerabilidad, es posible elaborar un mapa de riesgos. Hay varias formas de integrar la información. Una de ellas es hacer una matriz con colores que indiquen los niveles de amenaza y de vulnerabilidad.

Otros modos de integrar ambos mapas van desde lo más sencillo, sumando o multiplicando las clases, hasta lo más complejo, mediante índices multicriterio en el caso de los índices (cuadro 3).

$$
\text { Mapa de riesgo }=\text { amenaza }+ \text { vulnerabilidad }
$$




\section{A manera de conclusiones}

Al final del evento se realizó una mesa de conclusiones para subrayar los aspectos más importantes del seminario. Entre las conclusiones principales se citan las siguientes:

- De acuerdo con los estudios hidrogeológicos, se tiene evidencia que la PY no es precisamente una "gran reserva de agua", éste es sólo un mito que es necesario romper.

- Se reconoció que en la PY se tiene una gran cantidad y calidad de trabajos de disciplinas diferentes.

- El siguiente paso que es menester dar a corto plazo es la integración de estos estudios, para una mejor comprensión de la problemática y así ofrecer soluciones para la toma de decisiones.

- El sector gubernamental reconoce que se debe fomentar la vinculación entre la academia, el gobierno, el sector privado y la sociedad, mediante la formación de mesas de trabajo.

- También se enfatizó en la formación de nuevos recursos humanos, es decir, de jóvenes investigadores comprometidos con el trabajo en equipo transdisciplinario.

De esta manera, se dio fin a un acto académico que, más allá de ser un espacio para exponer nuestros trabajos, consideramos que cumplió una expectativa superior pues sirvió como plataforma para conocernos personalmente e identificarnos con nuestras áreas de trabajo. Sin embargo, pensamos que el trabajo en equipo aún debe continuar. A pesar de que cada persona se encuentra laborando en alguna universidad, centro de investigación o secretaría de gobierno, debemos permanecer en constante comunicación para el intercambio de información y colaboración mediante proyectos.

Hoy en día, están en marcha nuevas iniciativas de investigación para ampliar el ámbito del medio ambiente de las ciencias de la Tierra. Recientemente, la Unión Europea implementó una iniciativa para crear una taxonomía que comprende los suelos, el regolito y las aguas subterráneas como un organismo natural integrado que sustenta la vida en la Tierra; esta iniciativa es de suma importancia para la PY ya que sólo así, de forma integral, es posible generarla. 
Amenazas, vulnerabilidad y riesgo de

contaminación de las aguas subterráneas

en la Península de Yucatán

\section{Agradecimientos}

Al comité organizador del seminario "Análisis de la vulnerabilidad y riesgo de contaminación de las aguas subterráneas en la Península de Yucatán”, doctoras Patricia Quintana, Julia Pacheco y Diana Cabañas.Al Gobierno del Estado de Yucatán y al Consejo Nacional de Ciencia y Tecnología (Conacyt) por el apoyo al proyecto YUC-2006-C05-66I59, y al Conacyt por el financiamiento al proyecto 0903 I 5.A los comentarios de los árbitros anónimos por sus valiosas observaciones al manuscrito.

\section{FUENTES CONSULTADAS}

Bautista, F., (2008). "El conocimiento agrícola de los mayas en las zonas de karst”.Mi+d [en línea]. Disponible en: http://weblogs.madrimasd.org/ universo/archive/2008/02/0I/83655.aspx [2008, I5 de noviembre]. eY.Aguilar (2006). Proyecto "Análisis de la vulnerabilidad y riesgo de contaminación de las aguas subterráneas en Yucatán”. Financiado por el Consejo Nacional de Ciencia y Tecnología (Conacyt) y el Gobierno del Estado de Yucatán a través del fideicomiso Fondos Mixtos YUC2006-C05-66I57, 12 p.

C. Durán de Bazúa y R. Lozano (2000). "Cambios químicos en el suelo por aplicación de materia orgánica soluble tipo vinazas”. Revista Internacional de Contaminación Ambiental 16 (3), 89-I0I.

D. Bautista y C. Delgado-Carranza (2009). "Calibration of the Equations of Hargreaves andThornthwaite to Estimate the Potential Evapotranspiration in Semi-arid and Subhumid Tropical Climates for Regional Applications”. Atmósfera, 22 (4), 33 I-348.

_., et al. (2005). "Integración del conocimiento actual sobre los paisajes geomorfológicos de la Península de Yucatán”, en F. Bautista y G. Palacio (eds.). Caracterización y manejo de los suelos de la Península de Yucatán. Implicaciones agropecuarias, forestales y ambientales. México: Universidad Autónoma de Campeche (UAC)/Universidad Autónoma de Yucatán (UADY)/Instituto Nacional de Ecología (INE), 33-58.

,P.Quintana,Y.Aguilar,J. Pacheco y D. Cabañas (2009). Seminario “Análisis de la vulnerabilidad y riesgo de contaminación de las aguas subterráneas en la Península de Yucatán". I0 y II de diciembre de 2009. Mérida, Yucatán: Centro de Investigación y de Estudios Avanzados del Instituto Politécnico Nacional (Cinvestav-IPN), Unidad Mérida, 35 p.

Bocco, G., M. Mendoza y A.Velázquez (200I). "Remote Sensing and GIS-based 
Regional Geomorphological Zapping -a Tool for Land Use Planning in Developing Countries”. Geomorphology, 39, 211 -219.

Borges,A.C.y E.M. Mendiondo (2007).“Comparação entre equações empíricas para estimativa da evapotranspiração de referência na Bacia do Rio Jacupiranga". Revista Brasileira de Engenharia Agrícola e Ambiental, I I, 293-300.

Bouma,J. (1989).“Using Soil Survey Data for Quantitative Land Evaluation”, en B.A. Stewart (ed.). Advances in Soil Science, 9, I77-2I 3. (2006). "Hydropedology as a Powerful Tool for Environmental Policy Research”. Geoderma, I 3 I, 275-286.

Cabañas, D. y M.Vargas (2009). "Biosorción de colorantes en aguas residuales utilizando cáscaras de naranja", en F. Bautista et al. (eds.). Libro de Resúmenes del Seminario "Análisis de la vulnerabilidad y riesgo de contaminación de las aguas subterráneas en la Península de Yucatán". Mérida, Yucatán: Centro de Investigaciones en Geografía AmbientalUniversidad Nacional Autónoma de México (CIGA-UNAM), 35 p.

Conagua (2008). Estadísticas del agua en México. México: Secretaría de Medio Ambiente y Recursos Naturales (Semarnat), 228 p.

Denizman, C. (2003). "Morphometric and Spatial Distribution Parameters of Karstic Depressions, Lower Suwannee River Basin, Florida”. Journal of Cave and Karst Studies, 65 (I), 29-35.

Díaz, E., M. Anaya-Romero y D. de la Rosa (2009). "Modelo de evaluación agroecológica de tierras: erosión y contaminación en el entorno MicroLElS”, en F. Bautista et al. (eds.). Libro de Resúmenes del Seminario "Análisis de la vulnerabilidad y riesgo de contaminación de las aguas subterráneas en la Península de Yucatán”. Mérida, Yucatán: CIGA-UNAM, $35 \mathrm{p}$.

Doerfliger, N., P.Y.Jeannin y F.Zwahlen (1999).“WaterVulnerability Assessment in Karst Environments: a New Method of Defining Protection Areas Using a Multi-attribute Approach and GIS Tools (EPIK Method)". Environmental Geology, 39 (2), I65-176.

EPA (2002). A Lexicon of Cave and Karst Terminology with Special Reference to Environmental Karst Hydrology. Washington, D. C.: United States Environmental Protection Agency, 214 p.

Escolero, O., A. Jaime y E. Domínguez-Mariani (2009). "Methodology for 
Delineating an Hydrogeological Reserve Zone", en F. Bautista et al. (eds.). Libro de Resúmenes del Seminario "Análisis de la vulnerabilidad y riesgo de contaminación de las aguas subterráneas en la Península de Yucatán”. Mérida,Yucatán: CIGA-UNAM, 35 p.

FAO (2006). World Reference Base for Soil Resources. 2 ${ }^{\mathrm{a}}$ ed. Roma: Food and Agriculture Organization of the United Nations (World Soil Resources Reports no. 103), 128 p.

,(1990). Guidelines for profile description. $3^{\mathrm{a}}$ ed. Roma.

Florea, L. (2005).“'Using State-wide GIS Data to Identify the Coincidence between Sinkholes and Geologic Structure". Journal of Cave and Karst Studies, 67 (2), I $20-124$.

Frausto, O.y T.Ihl (2008).“Mapa de formas exocársticas del norte de Quintana Roo”, en M.A. Gutiérrez-Aguirre y A. Cervantes-Martínez (comps.). Estudio geohidrológico del norte de Quintana Roo, México [en línea]. Chetumal: Universidad de Quintana Roo, 37-50. Disponible en: http://biblioteca.coqcyt.gob.mx/bvic/Captura/upload/ESTUDIOGEOHIDROLOGICO-LIBRO.pdf [2009, 4 de junio].

Gold, G., C. Metcalfe y K. Drouillard (2009). "Contaminantes tradicionales y emergentes. Una amenaza para el acuífero”, en F. Bautista et al. (eds.). Libro de Resúmenes del Seminario "Análisis de la vulnerabilidad y riesgo de contaminación de las aguas subterráneas en la Península de Yucatán”. Mérida, Yucatán: CIGA-UNAM, 35 p.

Goldscheider, N. (2005).“'Karst GroundwaterVulnerability Mapping:Application of a New Method in the Swabian Alb, Germany”. Hydrological Journal, 13, 555-564.

González-Herrera, R., I. Sánchez-y-Pinto y J. Gamboa-Vargas (2002). Groundwater-flow Modeling in the Yucatan Karstic Aquifer, Mexico". Hydrogeology Journal, 10, 539-552.

Ihl, T., et al. (2007). "Identification of Geodisasters in the State of Yucatan, Mexico”. Neues Jahrbuch für Geologie Paläontologie Abhandlungen, 246 (3), 299-3II.

Kueny, J.A. y M. J. Day (2002). “Designation of Protected Karstlands in Central America: a Regional Assessment". Journal of Cave and Karst Studies, 64 (3), I65-174.

Lobo, D., et al. (2004). Guía metodológica para la elaboración del mapa de zonas 
áridas, semiáridas y subhúmedas secas de América Latina y el Caribe [en línea]. Caracas, Venezuela: Centro del Agua para Zonas Áridas y Semiáridas de América Latina y el Caribe/Programa Hidrológico Internacional-Organización de las Naciones Unidas para la Educación, la Ciencia y la Cultura, 54 p. Disponible en: http://www.unesco.org. uy/phi/biblioteca/bitstream/ / 23456789/435/ I/0525.pdf

López Macías, R.,V. Cobos Gasca y J. Rendón von Osten (2009)."Evaluación de los niveles de hidrocarburos (alifáticos y aromáticos policíclicos) en el agua subterránea de la ciudad de Mérida,Yucatán”, en F. Bautista et al. (eds.). Libro de Resúmenes del Seminario "Análisis de la vulnerabilidad y riesgo de contaminación de las aguas subterráneas en la Península de Yucatán”. Mérida, Yucatán: CIGA-UNAM, 35 p.

Marín, L. E., J. G. Pacheco y R. Méndez (2004). "Hidrogeología de la Península de Yucatán”, en B. Jiménez y L. Marín (eds.). El agua en México vista desde la academia. México:Academia Mexicana de Ciencias, I59-I76. et al. (1990)."Hurricane Gilbert: Its Effects on the Aquifer in Northern Yucatan, Mexico”, en E. S. Simpson y J. M. Sharp (eds.). Selected Papers on Hydrogeology from the 28th International Geological Congress. Washington D. C., Hanover:Verlag Heinz Heise, I I I-I 28.

Matthes, L. (2008). "Analysis and Evaluation of Ground and Surface Water Quality and Groundwater Flow in the Northern Aquifer of Quintana Roo, Mexico", en M.A. Gutiérrez-Aguirre y A. Cervantes-Martínez (comps.). Estudio geohidrológico del norte de Quintana Roo, México. Chetumal: Universidad de Quintana Roo, 59-99.

Mendoza, M. E., et al. (2009). Análisis de la aptitud territorial. Una perspectiva biofisica. México: Semarnat/INE/CIGA-UNAM, I 4 I p.

Morales, L. (2009). “El análisis espacial en la evaluación de la vulnerabilidad”, en F. Bautista et al. (eds.). Libro de Resúmenes del Seminario "Análisis de la vulnerabilidad y riesgo de contaminación de las aguas subterráneas en la Península de Yucatán”. Mérida, Yucatán: CIGA-unAM, 35 p.

Nemes, A., W. J. Rawls e Y.A. Pachepsky (2005). “Influence of Organic Matter on the Estimation of Saturated Hydraulic Conductivity". Soil Science Society of America Journal, 69, I330-I 337.

Oliver, J. E. (1980).“Monthly Precipitation Distribution:A Comparative Index”. 
Professional Geographer, 32 (I), 300-309.

Ortiz, M.A. y A. P. Méndez (1999). "Escenarios de vulnerabilidad por ascenso del nivel del mar en la costa mexicana del Golfo de México y el Mar Caribe”. Investigaciones Geográficas, 39, 68-8I.

Pacheco, J. y A. Cabrera (2009). "Vulnerabilidad y riesgo de contaminación por nitratos en aguas subterráneas del estado de Yucatán”, en F. Bautista et al. (eds.). Libro de Resúmenes del Seminario "Análisis de la vulnerabilidad y riesgo de contaminación de las aguas subterráneas en la Península de Yucatán”. Mérida, Yucatán: CIGA-UNAM, 35 p.

Pachepsky,Y.A.,W.J. Rawls y H.S. Lin (2006)."Hydropedology and Pedotransfer Functions". Geoderma, I3 I, 308-3 I6.

Perry, E., G.Velázquez-Olimán y L. Marín (2002). "The Hydrogeochemistry of the KarstAquifer System of the Northern Yucatan Peninsula, Mexico". International Geology Review, 44, I 2 I-22I.

, et al. (1995). "The Ring of Cenotes (Sinkholes) Northwest Yucatan, Mexico: Its Hydrogeologic Characteristics and Association with the Chicxulub Impact Crater". Geology, 23, I7-20.

—, et al. (2009). "Groundwater Geochemistry of the Yucatan Peninsula, Mexico: Constraints on Stratigraphy and Hydrogeology". Journal of Hydrology, 367, 27-40.

Rosa, D. de la, et al. (1996). CDMm Base de Datos Climáticos Mensuales [en línea]. Sevilla: Instituto de Recursos Naturales y Agrobiología-Consejo Superior de Investigaciones Científicas. Disponible en: http://www. evenor-tech.com/microleis/microlei/microlei2.aspx [2010, 19 de marzo].

Shofner, G.A., H. H. Mills y J.Duke (200I).“A Simple Map Index of Karstification and Its Relationship to Sinkhole and Cave Distribution in Tennessee". Journal of Cave and Karst Studies, 63 (2), 67-75.

Sobieraj, J. A., H. Elsenbeer y R. A. Vertessy (200I). "Pedotransfer Functions for Estimating Saturated Hydraulic Conductivity: Implications for Modeling Storm Flow Generation”.Journal of Hydrology, 25 I , 202-220.

Villasuso, P. M., et al. (2009). "La vulnerabilidad del acuífero cárstico costero del estado de Yucatán: ¿mito o realidad?”, en F. Bautista et al. (eds.). Libro de Resúmenes del Seminario "Análisis de la vulnerabilidad y riesgo de contaminación de las aguas subterráneas en la Península de Yucatán”. 
Mérida, Yucatán: CIGA-UNAM, 35 p.

Waele, J. de, L. Plan y P.Audra (2009). "Recent Developments in Surface and Subsurface Karst Geomorphology: an Introduction". Journal of Geomorphology, 106, I-8.

White, E. L. y W. B. White (1979). "Quantitative Morphology of Landforms in Carbonate Rock Basins in the Appalachian Highlands". Geological Society of America Bulletin, 90 (4), 385-396.

Wösten, J. H. M. (2002). “Pedotransfer Functions”, en R. Lal (ed.). Encyclopedia of soil science. Nueva York: M. Dekker, 967-97I.

—,Y.A. Pachepsky y W.J. Rawls (200I)."Pedotransfer Functions: Bridging the Gap Between Available Basic Soil Data and Missing Soil Hydraulic Characteristics". Journal of Hydrology, 25I, I23-I50.

—, et al. (1999). "Development and Use of a Database of Hydraulic Properties of European Soils". Geoderma, 90, 169-185.

Xu, C-Y. y D. Chen (2005). "Comparison of Seven Models for Estimation of Evapotranspiration and Groundwater Recharge Using Lysimeter Measurement Data in Germany". Hydrological Processes, 19,37I7-3734. yV.P.Singh (2005)." Evaluation of Three Complementary Relationship Evapotranspiration Models by Waters Balance Approach to Estimate Actual Regional Evapotranspiration in Different Climatic Regions”. Journal of Hydrology, 308, I05-121. 